\title{
CHARACTERISTICS OF STOCHASTIC RESONANCE IN ASYMMETRIC DUFFING OSCILLATOR
}

\author{
S. ARATHI and S. RAJASEKAR* \\ School of Physics, Bharathidasan University, \\ Tiruchirapalli 620 024, India \\ *rajasekar@cnld.bdu.ac.in \\ J. KURTHS \\ Postdam Institute for Climate Impact Research, \\ Haus No. A31, Postdam 14469, Germany
}

Received September 6, 2010; Revised January 11, 2011

\begin{abstract}
We study the characteristics of stochastic resonance (SR) in the Duffing oscillator with three types of asymmetries in its double-well potential. The asymmetries controlled by a parameter $\alpha$ are introduced in the potential by varying (i) the depth of the left-well alone, (ii) the location of the minimum of the left-well alone and (iii) both depth and location of the minimum of the left-well alone. The characteristics of SR in the asymmetric cases are different from the symmetric case $(\alpha=1)$. We find that asymmetry has a strong influence on the optimum noise intensity at which signal-to-noise ratio (SNR) is maximum, mean residence time at resonance and the probability distribution of residence time in the left- and right-wells. For a range of values of $\alpha, \alpha \neq 1$, SNR is found to be relatively higher than for $\alpha=1$.
\end{abstract}

Keywords: Stochastic resonance; asymmetric Duffing oscillator; signal-to-noise ratio; mean residence time.

\section{Introduction}

Features of stochastic resonance (SR) were often studied in bistable systems [Jung, 1993; Gammaitoni et al., 1998]; however, SR is also realized in monostable oscillators [Stokes et al., 1993; Evstigneev et al., 2004], chaotic systems [Gomes et al., 2003] and time-delay systems [Masoller, 2002; Borromeo \& Marchesoni, 2007]. Most of the analysis of SR are on symmetric double-well potential systems. Since it is difficult to preserve symmetry in real physical or even natural systems, the study of asymmetric systems has received considerable interest. For example, the occurrence of SR has been studied in an overdamped asymmetric doublewell system (where asymmetry is introduced by adding a constant bias which changes both depth and location of the two wells) [Gammaitoni et al., 1989] and Schmitt trigger [Marchesoni et al., 1999]. McNamara and Wiesenfeld [McNamara \& Wiesenfeld, 1989] developed a discrete two-state theory for understanding SR in overdamped symmetric bistable systems. With this theory, an analytical expression for signal-to-noise ratio (SNR) can be obtained. The two-state theory has been applied to an overdamped asymmetric system where depth and location of the minima of the two wells are altered [Li, 2002; Wio \& Bouzat, 1999]. The variation of SNR with the frequency of the external periodic force in the bistable potential $a x^{2}+b x^{3}+c x^{4}$ was reported [So \& Liu, 2002]. Improving the measurement conditions by stochastic resonance is demonstrated in an experiment with 
optomechanically coupled oscillator [Mueller et al., 2009]. Double SR was found in an asymmetric system recently [Borromeo \& Marchesoni, 2010]. Though there are studies on SR in asymmetric systems, the influence of asymmetries especially the difference in the depth of the wells and the location of the minima of the wells have not been explored systematically.

The aim of the present paper is to investigate the role of certain forms of asymmetries for the various characteristics of $\mathrm{SR}$ in the paradigmatic double-well Duffing oscillator. For this purpose, we consider the Duffing oscillator with three different asymmetric potentials $V_{1}, V_{2}$ and $V_{3}$ given by

$$
V_{i}(x)= \begin{cases}-\frac{1}{2} \omega_{0}^{2} x^{2}+\frac{1}{4} \beta x^{4}, & x \geq 0 \\ -\frac{1}{2} \omega_{0}^{2} A_{i} x^{2}+\frac{1}{4} \beta B_{i} x^{4}, & x<0\end{cases}
$$

where $i=1,2,3, A_{1}=B_{1}=\alpha, A_{2}=1 / \alpha^{2}, B_{2}=$ $1 / \alpha^{4}, A_{3}=1, B_{3}=1 / \alpha^{2}$ and $\alpha, \beta, \omega_{0}^{2}>0 . \alpha$ is here the parameter which controls the asymmetry. The potentials $V_{1}, V_{2}$ and $V_{3}$ are shown in Fig. 1. In all three potentials, the shape of the right-well is unaffected by $\alpha$. In the potential $V_{1}$, the depth of the left-well depends on $\alpha: \Delta V_{-}=$ $\alpha \omega_{0}^{4} /(4 \beta)$. The location of the left-well minimum, $x_{\min }^{\mathrm{L}}=-\sqrt{\omega_{0}^{2} / \beta}$, is independent of $\alpha$. In the potential $V_{2}$, the location of the left-well minimum is given by $x_{\text {min }}^{\mathrm{L}}=-\alpha \sqrt{\omega_{0}^{2} / \beta}$, which depends on $\alpha$ while the depth $\Delta V_{-}$remains as $\omega_{0}^{4} /(4 \beta)$. On the other hand, in the potential $V_{3}, x_{\min }^{\mathrm{L}}=-\alpha \sqrt{\omega_{0}^{2} / \beta}$ and $\Delta V_{-}=\alpha^{2} \omega_{0}^{4} /(4 \beta)$.

The asymmetric potentials $V_{1}, V_{2}$ and $V_{3}$ can be realized experimentally. For example, in the mechanical model of the Duffing oscillator described in [Moon \& Holmes, 1979], we can introduce the above types of asymmetries by simply varying the position of the left-side magnet and its strength. In submicron Bi-wires, modeled by a double-well potential, the applied magnetic field is varied to change the depth of one well [Zimmerman et al., 1991]. A double-well system with different depths is realized as an appropriate model of auditory nerve fibre response [Simiu, 2002].

The equations of motion of the Duffing oscillator with the potentials $V_{1}, V_{2}$, and $V_{3}$, linear damping, periodic force $f \sin \omega t$ and Gaussian white noise $\eta(t)$ are given by

$$
\ddot{x}+d \dot{x}+\frac{\mathrm{d} V_{i}}{\mathrm{~d} x}=f \sin \omega t+\eta(t), \quad i=1,2,3 .
$$

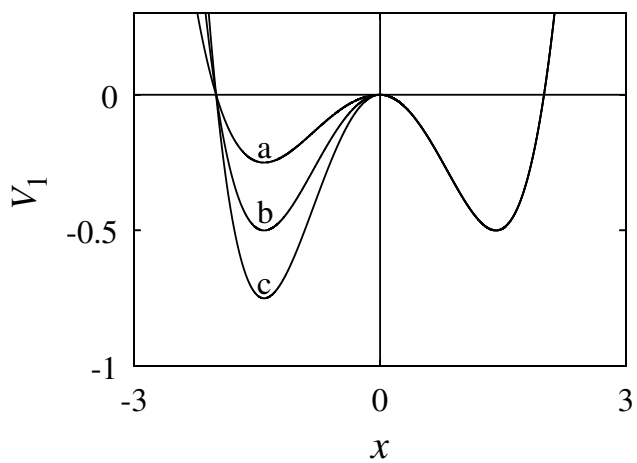

(a)

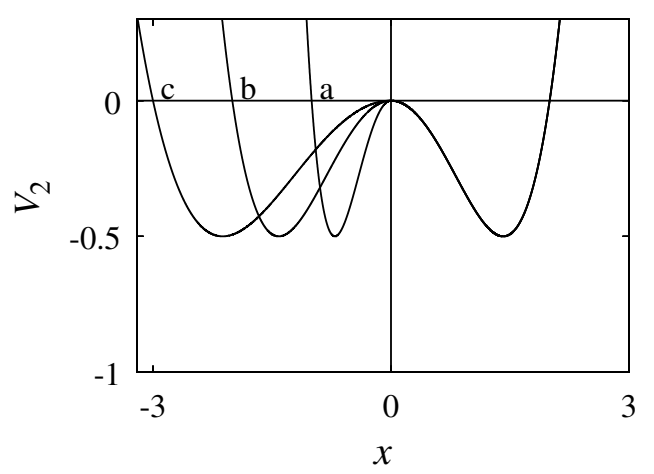

(b)

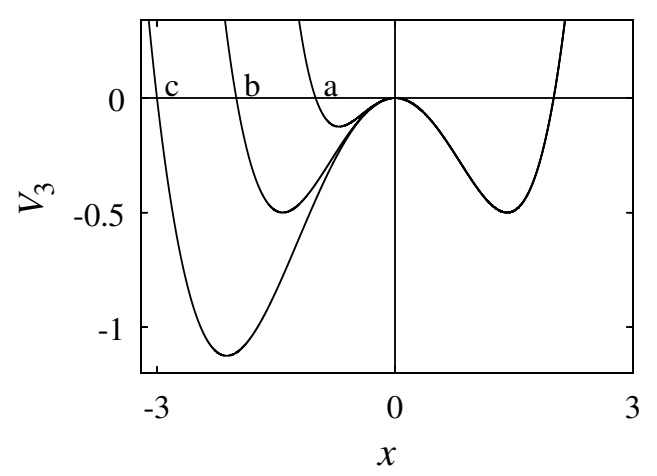

(c)

Fig. 1. The shape of the potentials $V_{1}, V_{2}$, and $V_{3}$ for $\omega_{0}^{2}=1, \beta=0.5$ and $\alpha=$ (a) 0.5 , (b) 1 and (c) 1.5 .

We call the systems with the asymmetric potentials $V_{1}, V_{2}$ and $V_{3}$ as systems $1-3$ respectively. $\eta(t)$ is chosen as Gaussian white noise with zero mean, unit variance and correlation $\langle\eta(t) \eta(s)\rangle=D \delta(t-s)$ where $D$ is the noise intensity.

In our study, we noticed several interesting differences on the features of SR in the three asymmetric systems compared to the symmetric system. The noise intensity $D_{\mathrm{MAX}}$ at which SNR is maximum 
(denoted as $\mathrm{SNR}_{\mathrm{MAX}}$ ) is relatively higher (lower) than that of the symmetric system $(\alpha=1)$ for $\alpha>1(\alpha<1)$. In the symmetric system the probability distribution of normalized residence times, $P\left(T_{\mathrm{R}} / T\right)$ where $T_{\mathrm{R}}$ represents residence time in a well and $T=2 \pi / \omega$, in the left- and right-wells are identical for all values of the noise intensity. In contrast to this, $P$ is different in two wells in the asymmetric cases. The parameter $\alpha$ has a strong influence on the mean residence time $T_{\mathrm{MR}}$ at resonance. In the symmetric case, $T_{\mathrm{MR}}$ in the left- and right-wells are $T / 2$ at resonance. This characteristic property is destroyed by the asymmetry. However, at resonance the sum of the mean residence times in the two wells is equal to the period of the external periodic force.

The paper is organized as follows. In Sec. 2, we show the occurrence of SR in the three systems for a set of values of asymmetric parameter $\alpha$. We compare the SR phenomenon in the systems with time-series plot, variation of $D_{\mathrm{MAX}}$ and SNR $\mathrm{MAX}$. In Sec. 3, we discuss the effect of asymmetries on the probability distribution of normalized residence time in the two wells and also on the variation of mean residence time at resonance. Section 4 contains concluding remarks.

\section{Effect of Asymmetries on SNR and Time-Series Plot}

We fix the parameters in Eq. (2) as $d=0.5, \omega_{0}^{2}=1$, $\beta=0.5, \omega=0.05$ and $f=0.38$. In all three noisefree systems for any fixed value of $\alpha, \alpha>0$, crosswell motion is not possible for $f=0.38$. Before studying the noise-induced resonance dynamics, we consider the noise-free system. We computed the variation of output signal amplitude $S$ as a function of $\omega$ and $\alpha$ in the absence of noise. For a range of fixed values of $\alpha$ when $\omega$ is varied from a small value we found typical nonlinear resonance in these systems. $S$ is maximum at a critical value of $\omega$ denoted as $\omega_{\max }$. This critical value of $\omega$ varies with $\alpha$. Figure 2(a) shows $S$ versus $\omega$ for $\alpha=1.5$ and $\alpha=1$ for the systems 1-3 and corresponding to the orbit lying in the left-well. $\omega_{\max }$ is different for the three systems. $S_{\max }$ of systems 2 and 3 are relatively higher than that of system 1 . This is because for $\alpha=1.5$ the distance between the left-equilibrium point and the middle equilibrium point (origin) of systems 2 and 3 is greater than that of system 1. In all these systems, for each fixed value of $\alpha$ in the range $[0,2]$ when $\omega$ is varied there is no cross-well

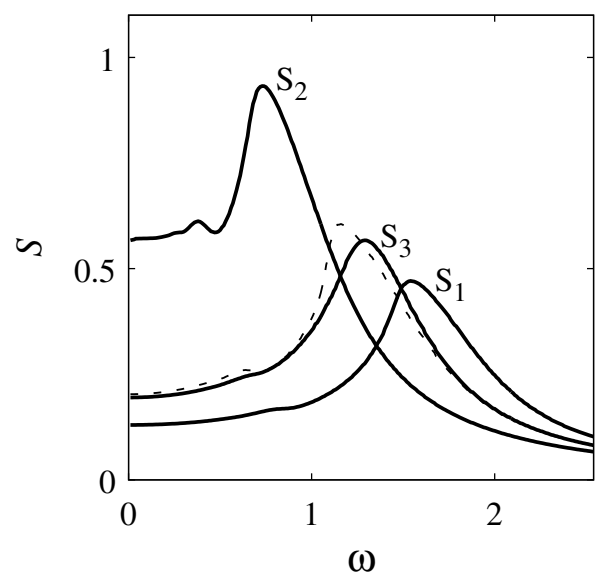

(a)

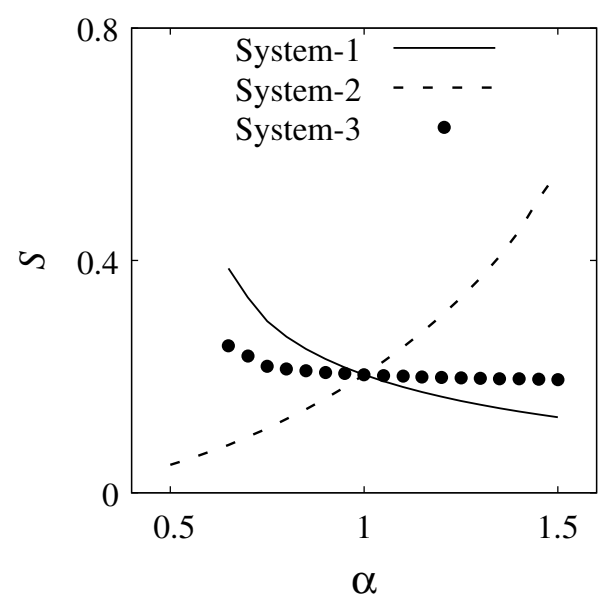

(b)

Fig. 2. (a) Frequency-response curves of systems 1-3 denoted as $S_{1}, S_{2}$ and $S_{3}$ respectively for $\alpha=1.5$ and $D=0$. The dashed curve represents the response amplitude $S$ of the symmetric system $(\alpha=1)$. The values of other parameters in the noise-free systems are $\omega_{0}^{2}=1, \beta=0.5$ and $f=0.38$. (b) The amplitude $S$ of the orbit in the left-well for a range of values of $\alpha$ for the three systems with the particle initially in the left-well for each value of $\alpha$ for $\omega=0.05$ and $D=0$.

motion at resonance. Further, for small values of $\alpha$ and $\omega$ there is no orbit confined to the left-well alone for systems 1 and 3 where the depth of the left-well is changing with $\alpha$. Trajectories starting from the left-well with different initial conditions cross the potential barrier and jump to the right-well. After the transient motion the orbit is confined to the right-well alone (shape of the right-well is independent of $\alpha$ ). In Fig. 2(b) we plotted $S$ versus $\alpha$ for the three systems in the absence of external noise $\eta(t)$. In systems 1 and 3 the amplitude $S$ of the output 
signal decreases with $\alpha$. In contrast to this, in system 2 the amplitude $S$ increases with $\alpha$ and this is because of increase in the distance between the leftwell equilibrium point and widening of the left-well potential. In any case in the three systems there is no resonance and no cross-well motion when $\alpha$ is varied in the absence of noise.

Now, we consider systems $1-3$ in the presence of external noise. We fix the value of $\omega$ as 0.05 . Equation (2) is integrated numerically with the fourth-order Runge-Kutta method from $t$ to $t+\Delta t$ without noise term and then noise is added to the state variable $x$ as $x(t+\Delta t) \rightarrow x(t+\Delta t)+\sqrt{D \Delta t} \xi$ where $\xi$ is a Gaussian random number generated using Box-Muller algorithm. This is repeated in each integration step $\Delta t$. To characterize $\mathrm{SR}$ we numerically calculated SNR from the power spectral density estimated using fast Fourier transform (FFT) with $n=2^{12}$ data with sampling interval $\tau$. Average power spectrum of 25 realizations of Gaussian random numbers is considered to have a better accuracy. SNR can be defined in units of decibel as

$$
\mathrm{SNR}=10 \log _{10}\left(\frac{S}{N}\right) \mathrm{dB}
$$

For $\alpha=1$, we numerically intergrated the system with three different time steps. For each case the critical value of $D$ (denoted as $D_{\mathrm{MAX}}$ ) at which SNR is maximum is calculated. For $\Delta t=0.01,0.005$ and 0.001 the values of $D_{\mathrm{MAX}}$ are found to be $0.15010,0.15105$ and 0.15076 . Since the magnitude of the relative error with $\Delta t=0.01$ is less than $1 \%$ we use this time step for the numerical simulation.

Figures 3(a)-3(c) show the variation of SNR versus $D$, for each fixed value of $\alpha$, as $D$ increases from a small value the SNR value increases and attains a maximum at a value of $D$, say, $D_{\text {MAX }}$ and decreases with further increase in $D$. This is the typical characteristic of SR. In Fig. 4, we plotted $D_{\text {MAX }}$ at which SNR is maximum (represented as $\left.\mathrm{SNR}_{\mathrm{MAX}}\right)$. In all three systems $D_{\mathrm{MAX}}$ $(\alpha<1)$ decreases (increases) when $\alpha$ is decreased (increased) from the value 1 . That is, SR can be observed at relatively lower noise intensity than that of the symmetric double-well system by reducing $\Delta V_{-}$alone or $x_{\min }^{\mathrm{L}}$ alone or both. For $\alpha>1$, $D_{\text {MAX }}$ increases rapidly with $\alpha$ in systems 2 and 3 . But in system 1 very slow variation of $D_{\text {MAX }}$ is noticed for $\alpha>1$. Since the left-well barrier height increases with $\alpha$, sufficiently large noise intensity

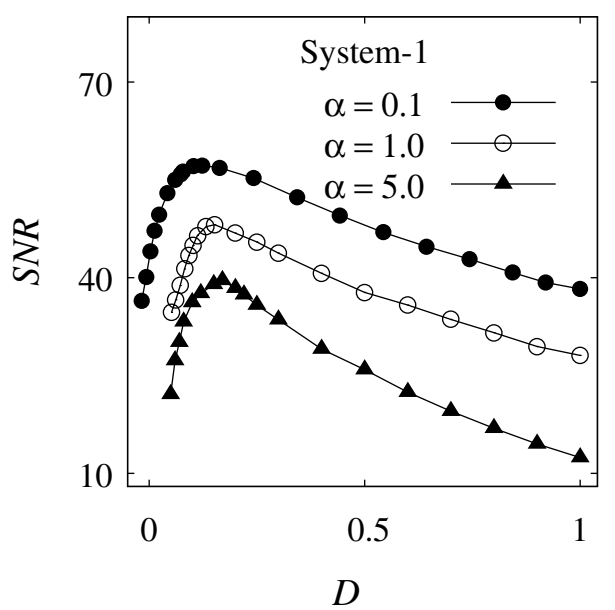

(a)

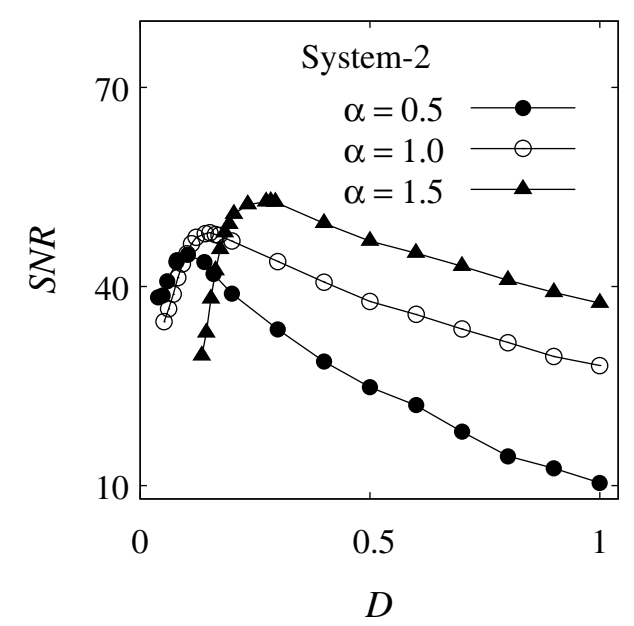

(b)

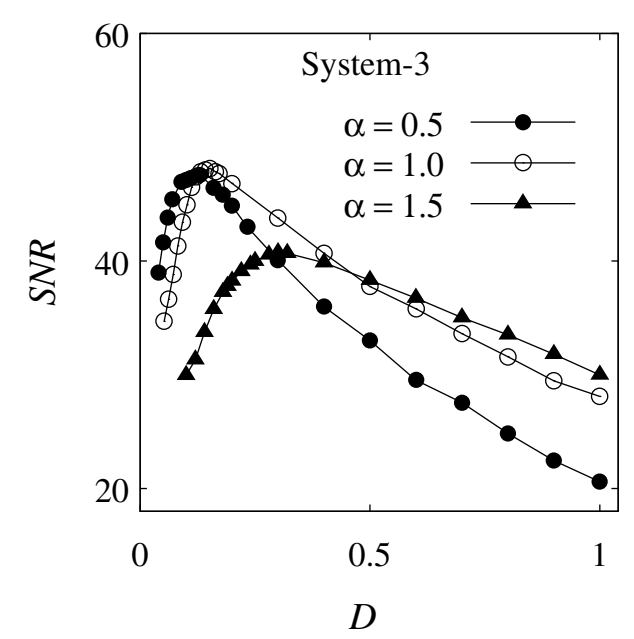

(c)

Fig. 3. (a)-(c) Variation of SNR as a function of the noise intensity $D$ of systems $1-3$ for three fixed values of $\alpha . \alpha=1$ corresponds to the symmetric double-well system. 


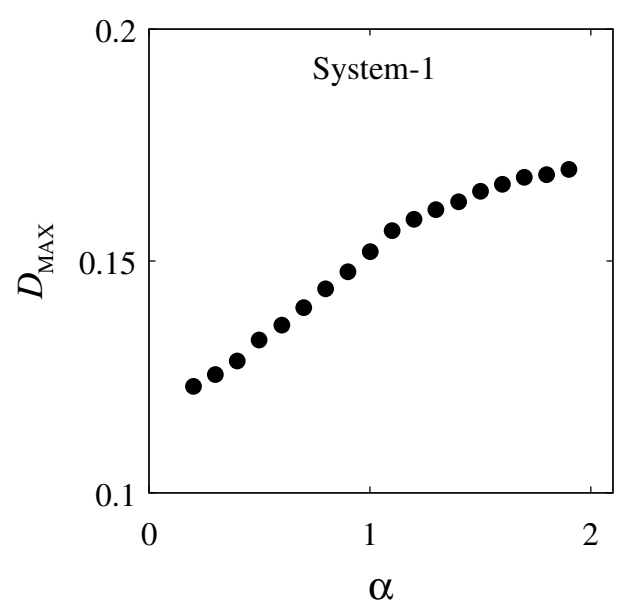

(a)

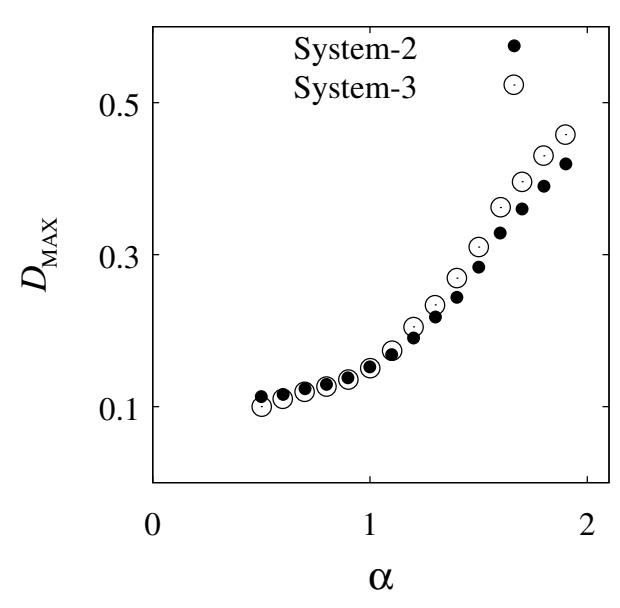

(b)

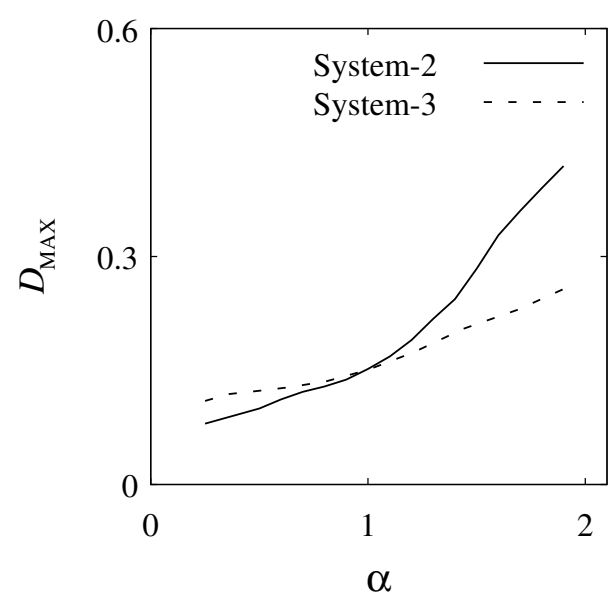

(c)

Fig. 4. (a) and (b) Plot of $D_{\mathrm{MAX}}$ (at which SNR is maximum) as a function of $\alpha$ for systems 1-3 respectively. (c) Plot of numerically computed $D_{\mathrm{MAX}}$ versus $\alpha$ for system 2 (continuous line) and system 3 (dashed line) with $A_{2}=1 / \alpha^{2}$, $B_{2}=1 / \alpha^{4}, A_{3}=1$ and $B_{3}=1 / \alpha^{4}$. is needed for the particle to move from left-well to right-well. This is the reason for increase in $D_{\mathrm{MAX}}$ with $\alpha$ in system 1 . In system 2 the location of minimum of the left-well increases with increase in $\alpha$ so the particle covers a wide area in the left-well. Consequently, higher noise intensity is required to push the particle from left-well to right-well for large values of $\alpha$. In system 3, $D_{\text {MAX }}$ increases because of the increase in both depth and location of the minimum of the left-well with $\alpha$. Considering the result from Fig. 4(b) we find that $D_{\text {MAX }}$ for systems 2 and 3 are almost the same. In this connection we note that in Eq. (1), in order to have the same expression for $x_{\mathrm{L}}^{\mathrm{min}}$ in systems 2 and 3 , $B_{3}$ is chosen as $1 / \alpha^{4}$. However, the depth of the left-well potential of system 2 is independent of $\alpha$ while that of system 3 is $\Delta V_{-}=\alpha^{2} \omega_{0}^{2} /(4 \beta)$. For clarity, in Fig. $4(\mathrm{c})$ we plotted $D_{\mathrm{MAX}}$ versus $\alpha$ for systems 2 and 3 with $A_{2}=1 / \alpha^{2}, B_{2}=$ $1 / \alpha^{4}, A_{3}=1$ while $B_{3}=1 / \alpha^{4}$. In this case $D_{\text {MAX }}$ of two systems are widely different for a range of values of $\alpha$.

Next, we obtain different changes of $\mathrm{SNR}_{\mathrm{MAX}}$ in dependence on $\alpha$ (Fig. 5). In systems 1 and 3, $\mathrm{SNR}_{\text {MAX }}$ decreases and in system 2 , it increases with increase in $\alpha$. That is, SNR can be improved (compared to the symmetric system) either by increasing $x_{\min }^{L}$ or by decreasing $\Delta V_{-}$or both. It is important to note that though $D_{\mathrm{MAX}}(\alpha<1)$ is lower than $D_{\operatorname{MAX}}(\alpha=1)$ in all three asymmetric systems, $\operatorname{SNR}_{\text {MAX }}(\alpha<1)<\operatorname{SNR}_{\text {MAX }}(\alpha=1)$ happens only in system 2 . The different variations of SNR $_{\text {MAX }}$ found in Fig. 5, can be accounted from the effect of $\alpha$ on the amplitude $S$ of the orbit in

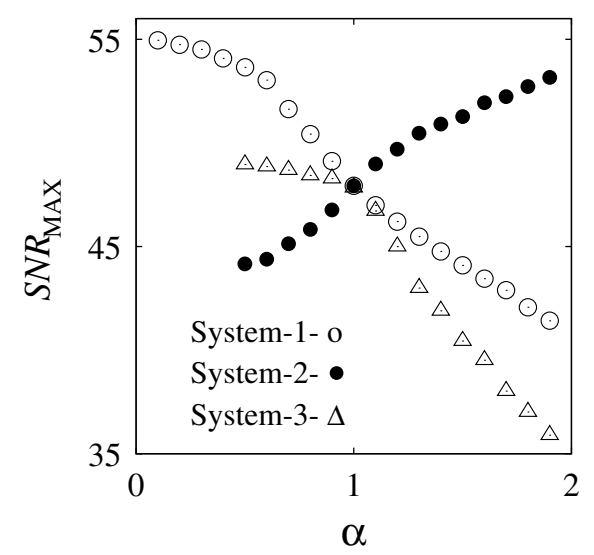

Fig. 5. Variation of $\mathrm{SNR}_{\mathrm{MAX}}$ as a function of $\alpha$ for systems $1-3$. For $\alpha=1$ the three systems become the symmetric double-well system. 


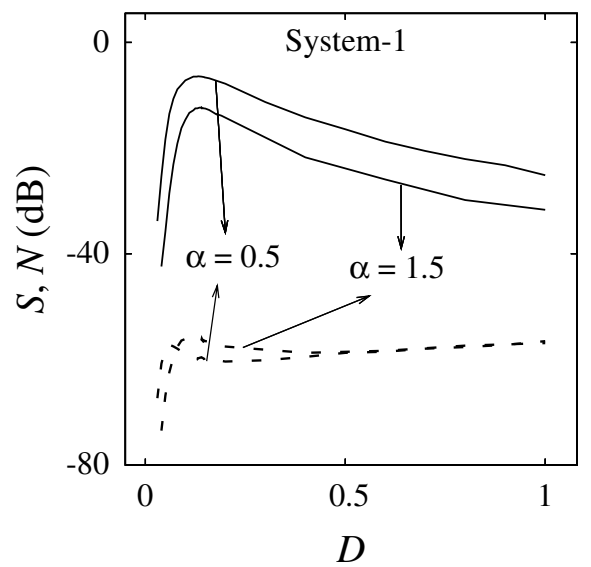

(a)

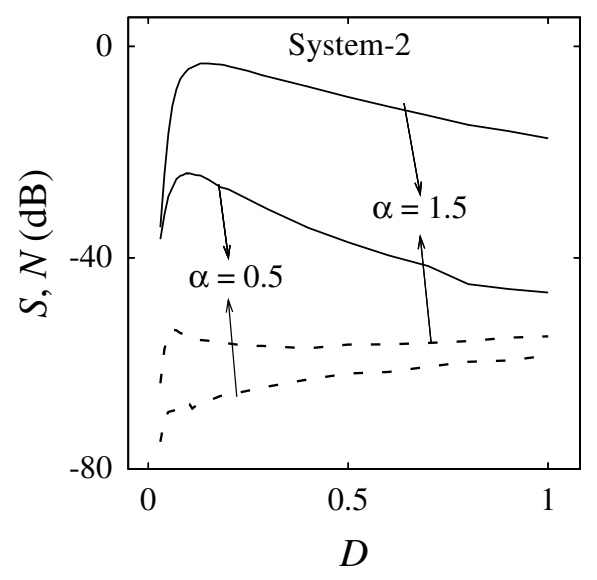

(b)

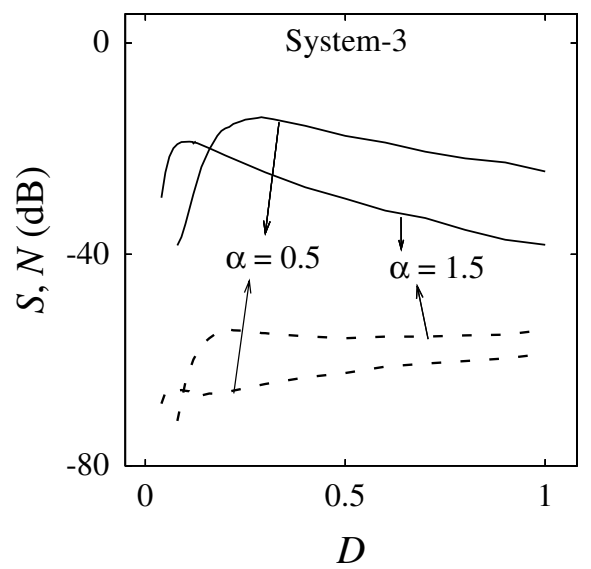

(c)

Fig. 6. (a)-(c) Variation of the amplitude of the output signal (represented by continuous lines) and the noise background (represented by dashed lines) of systems 1-3 respectively for two values of $\alpha=0.5$ and 1.5 for a range of values of the noise intensity $D$. The values of the parameters are $\omega_{0}^{2}=1, \beta=0.5, f=0.38$ and $\omega=0.05$. left-well in the absence of noise and the variation of the amplitude of the orbit and noise background in the noise driven systems. In Fig. 2(b), the amplitude $(S)$ of the orbit in the left-well in the absence of noise decreases with increase in $\alpha$ in systems 1 and 3 but increases in system 2 . Figures $6(\mathrm{a})-6(\mathrm{c})$ show the variation of $S$ and $N$ of systems $1-3$ for two values of $\alpha$ for a range of noise intensity $D$. It is noted that SNR is not maximum at the value of $D$ at which $S$ becomes maximum. For example, in system 1 for $\alpha=0.1,1$, and $5, S$ is maximum at $D=0.175,0.15$ and 0.125 respectively while SNR is maximum at $D=0.123,0.152$ and 0.171 , respectively. As pointed out in [Gammaitoni et al., 1989] the amplitude is an independent measure of SR. $S$ decreases with increase in $\alpha$ in systems 1 and 3 and increases with $\alpha$ in system 2 not only in the absence of noise [Fig. 2(b)] but also in the presence of noise (as shown in Figs. 6(a)-6(c) for two values of $\alpha$ ). In system 1 for a wide range of values of $D$ including $D_{\mathrm{MAX}}$, we notice $S(\alpha=1.5, D)<S(\alpha=$ $0.5, D)$ while $N(\alpha=1.5, D)>N(\alpha=0.5, D)$. That is, for a fixed value of $D, S(N)$ decreases (increases) with $\alpha$. Therefore, $\mathrm{SNR}_{\text {MAX }}$ decreases with $\alpha$. In system 2 the left-well potential widens [Fig. 1(b)] and both $S$ and $N$ increase with $\alpha$. However, the increase in $S$ is much higher than the increase in $N$. SNR MAX is thus found to increase with $\alpha$. In system 3, even though the left-well equilibrium point moves away from the origin with $\alpha$, the depth increases and the combined effect makes the signal amplitude of noise-free case to decrease. Consequently, as shown in Fig. 6(c) for a wide range of noise intensity $S\left(\alpha_{2}, D\right)<S\left(\alpha_{1}, D\right)$ while $N\left(\alpha_{2}, D\right)>N\left(\alpha_{1}, D\right)$ where $\alpha_{2}>\alpha_{1}$. The noise background also increases. Thus $\mathrm{SNR}_{\mathrm{MAX}}$ decreases with $\alpha$.

In the symmetric system at $D=D_{\mathrm{MAX}}$, almost periodic switching between both wells with period $T / 2$ occurs where $T=2 \pi / \omega$ is the period of the input signal $f \sin \omega t$. This characteristic property of SR is destroyed by the asymmetries. Figure 7 shows $x$ versus $t$ for the symmetric and asymmetric systems at $D=D_{\mathrm{MAX}}$. In Fig. 7(a), corresponding to the symmetric system $(\alpha=1)$, the trajectory spends almost $T / 2$ time duration in one-well before switching to the other well. In all asymmetric systems, for $\alpha<1$ the time spent in the region $x>0$ is relatively larger than in the region $x<0$, while the reverse effect is observed for $\alpha>1$ [Figs. 7(b) $-7(\mathrm{~g})]$. 


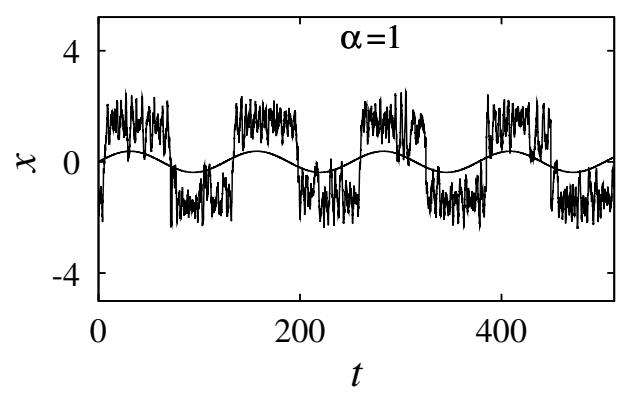

(a)

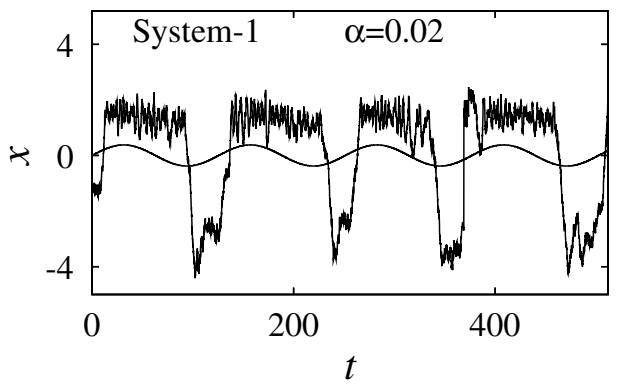

(b)

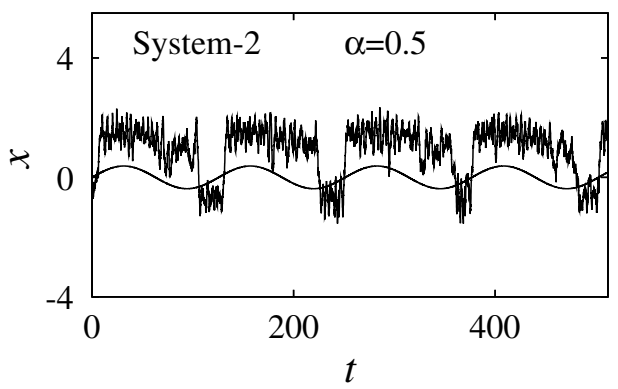

(d)

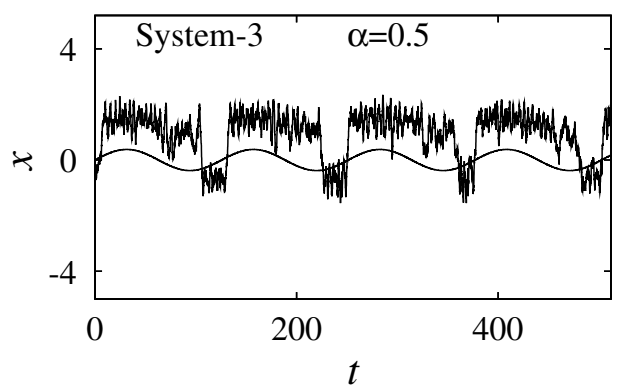

(f)

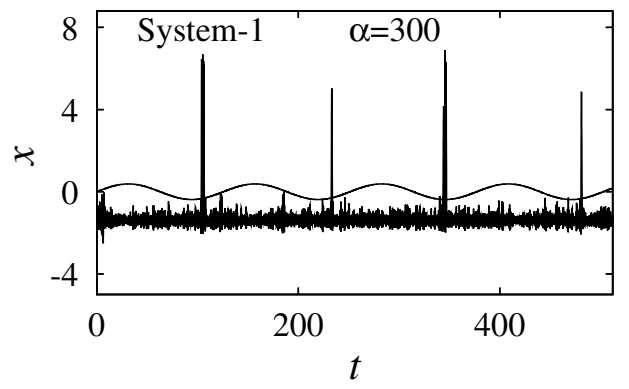

(c)

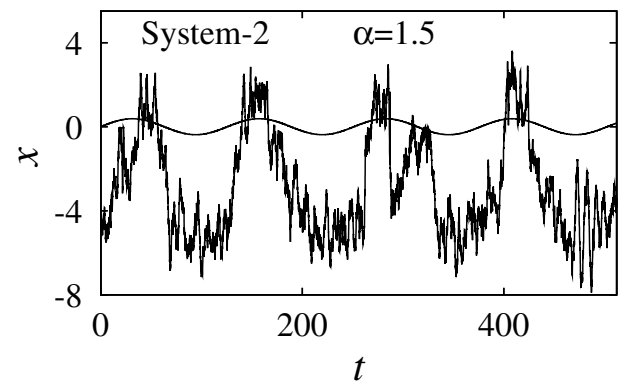

(e)

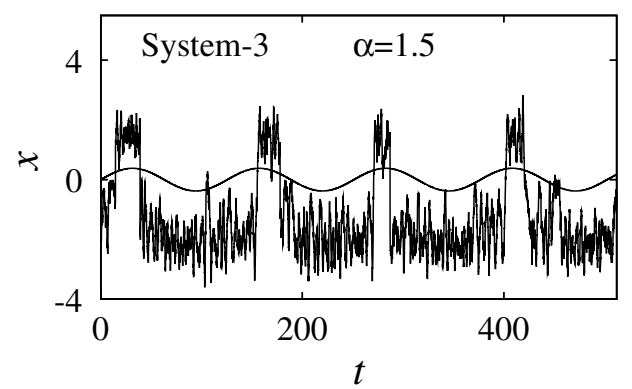

(g)

Fig. 7. Time-series plots of systems $1-3$ at $D_{\mathrm{MAX}}$, i.e. SNR is maximum. The $D_{\mathrm{MAX}}$ values are the following: (a) $\alpha=1$ (symmetric system), $D_{\mathrm{MAX}}=0.152$; (b) System $1, \alpha=0.02, D_{\mathrm{MAX}}=0.11$; (c) System $1, \alpha=300, D_{\mathrm{MAX}}=0.172$; (d) System 2, $\alpha=0.5, D_{\mathrm{MAX}}=0.096$; (e) System 2, $\alpha=1.5, D_{\mathrm{MAX}}=0.268$; (f) System $3, \alpha=0.5, D_{\mathrm{MAX}}=0.101$; (g) System $3, \alpha=1.5, D_{\mathrm{MAX}}=0.271$. 


\section{Effect of Asymmetries on the Probability Distribution of Normalized Residence Time and Mean Residence Time}

In this section we analyze the influence of asymmetries on the probability distribution of the normalized residence time and the mean residence time. The residence time $T_{R}$ in a well is defined as the duration of time the system resides in one well before switching to the other one. $T_{\mathrm{R}}$ of the left-well and the right-well are calculated over $10^{5}$ transitions from one well to another one. Then the normalized residence times $T_{\mathrm{R}} / T$ where $T=2 \pi / \omega$ are calculated. In Figs. 8-10 probability distributions of normalized residence time of left- and right-wells of the symmetric system $(\alpha=1)$ and of system 1 for $\alpha=0.02$ and 300 respectively for three values of noise intensity $D$ are given. The figures demonstrate the effect of $\alpha$ on $P\left(T_{\mathrm{R}} / T\right)$. When $\alpha=1$ (Fig. 8) the following features of $P\left(T_{\mathrm{R}} / T\right)$ are observed: (i) The $P\left(T_{\mathrm{R}} / T\right)$ of the left-well and the right-well are identical for any value of $D>D_{\mathrm{c}}$. (ii) For a value of $D$ just above $D_{\mathrm{c}}, P\left(T_{\mathrm{R}} / T\right)$ is very wide and exhibits a sequence of Gaussian-like peaks centered around $T_{\mathrm{R}} / T=n+1 / 2, n=0,1,2, \ldots$ i.e. about half integer multiples of period $T$ of the periodic driving force. (iii) As $D$ increases further, the range of $T_{\mathrm{R}} / T$ decreases and the $P\left(T_{\mathrm{R}} / T\right)$ profile moves towards the minimum value of $T_{\mathrm{R}} / T$. (iv) At $D=D_{\mathrm{MAX}}$, the $P\left(T_{\mathrm{R}} / T\right)$ of both left-well and right-well have a Gaussian peak centered at $T_{\mathrm{R}} / T=1 / 2$.

Compared to symmetric system in the asymmetric system we notice additional peaks at $T_{\mathrm{R}} / T=1,2,3, \ldots$ In the symmetric system the depths of the two wells at $t=n T, n=1,2, \ldots$ are the same and are modulated by the external force $f \sin \omega t$. Suppose, the particle is initially in the leftwell. It has higher chance to move to the right-well when the depth of the left-well is minimum. If the particle has crossed the left-well when its depth is about minimum then the right-well depth at this time $\left(t^{\prime}\right)$ is about maximum. Now the chance for the particle to switch from the right-well is higher when its depth becomes minimum which will happen when $t=t^{\prime}+(2 n+1) T / 2, n=0,1,2, \ldots$. Consequently, we expect peaks in $P\left(T_{\mathrm{R}} / T\right)$ at $T_{\mathrm{R}} / T=n+1 / 2, n=0,1,2, \ldots$ for both leftand right-wells. Like the symmetric system, in the asymmetric systems with $\alpha<1$ we expect peaks at $T_{\mathrm{R}} / T=n+1 / 2, n=0,1,2, \ldots$ In addition to these peaks in Fig. 9 for $D=0.08$, we notice peaks
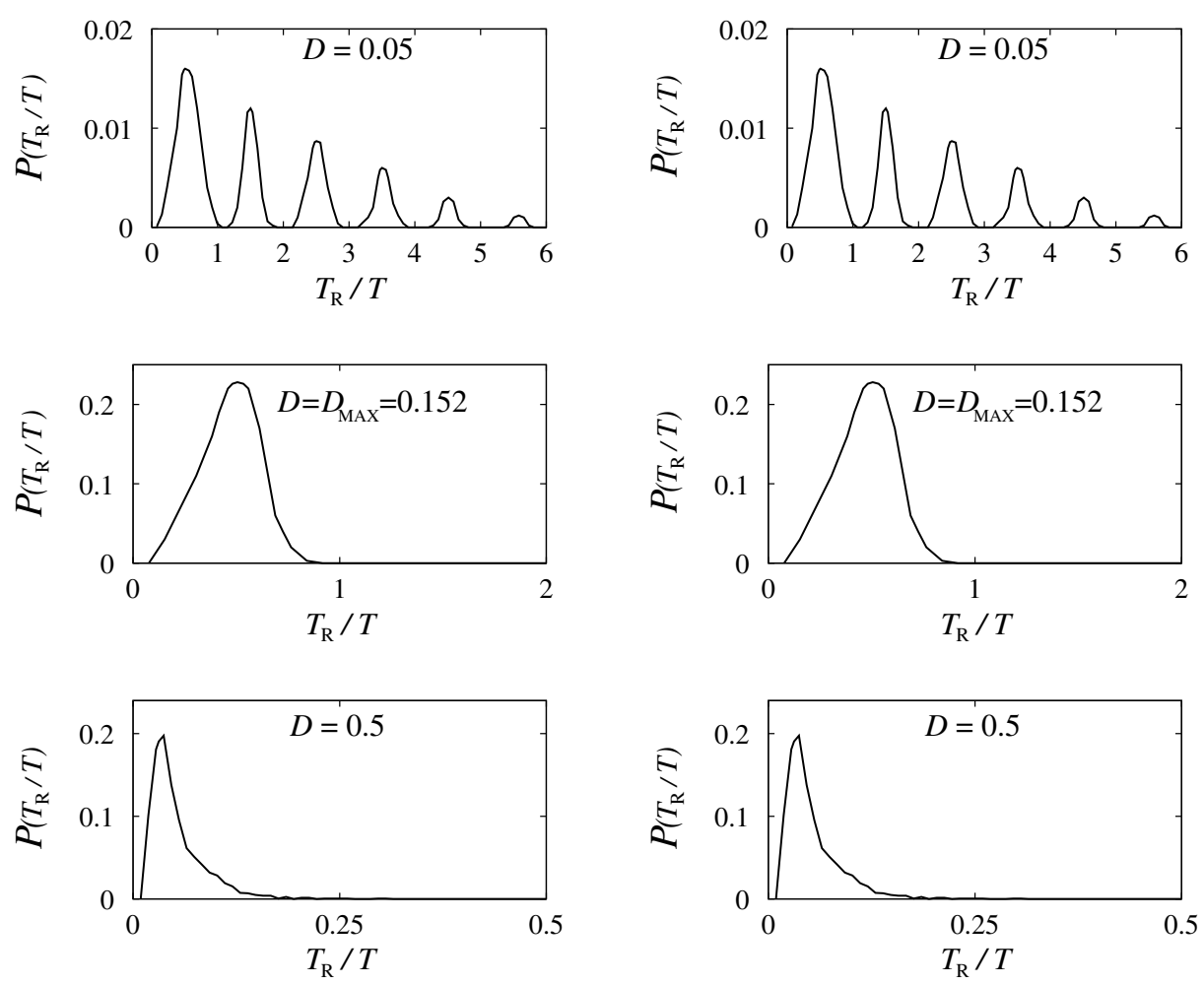

Fig. 8. Probability distribution of normalized residence times $P\left(T_{\mathrm{R}} / T\right)$ of the symmetric system $(\alpha=1)$ for three values of noise intensity $D$. The first column of subplots are for the left-well and the second column is for the right-well. 

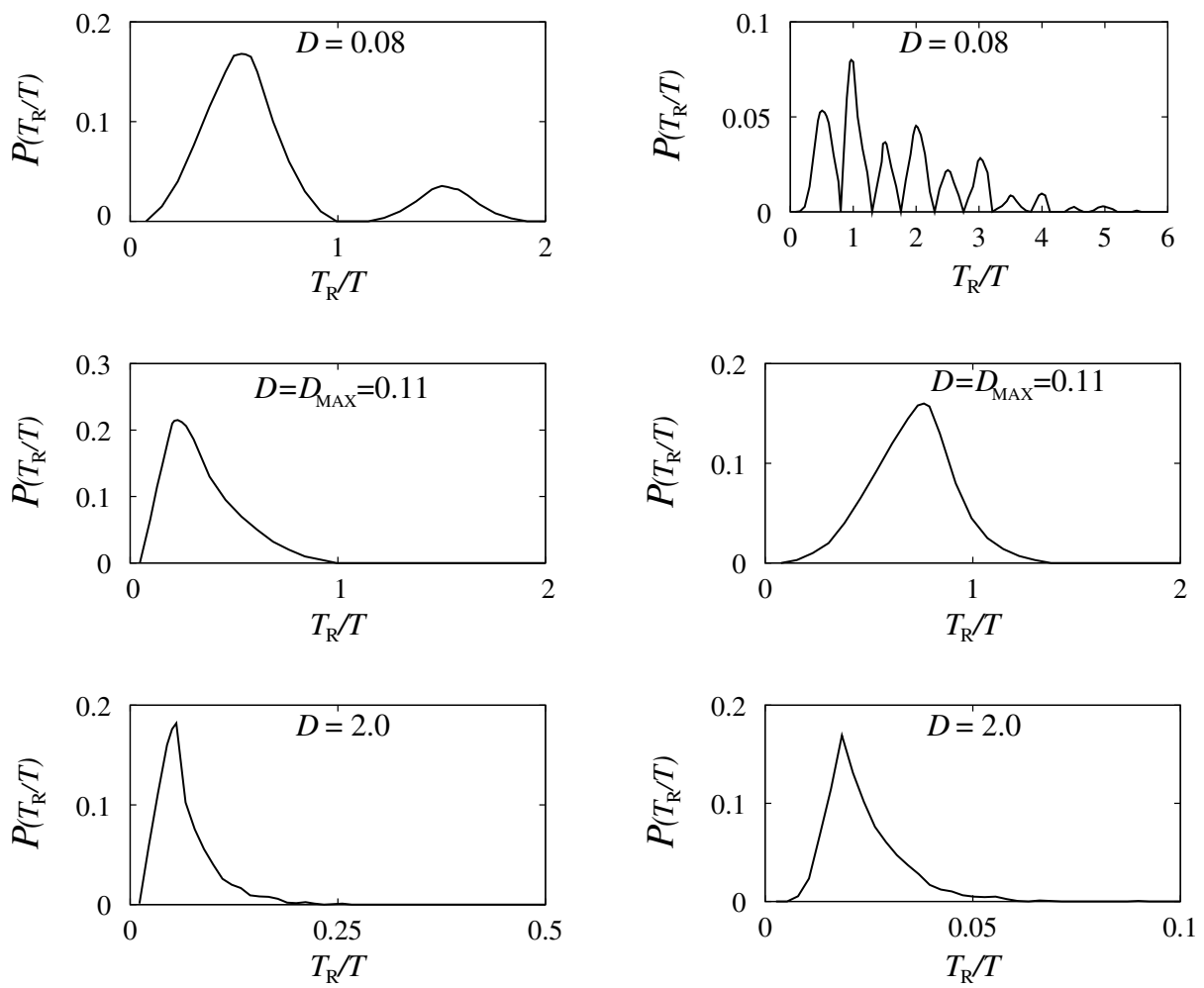

Fig. 9. Same as Fig. 8 but for the asymmetric system 1 with $\alpha=0.02$.
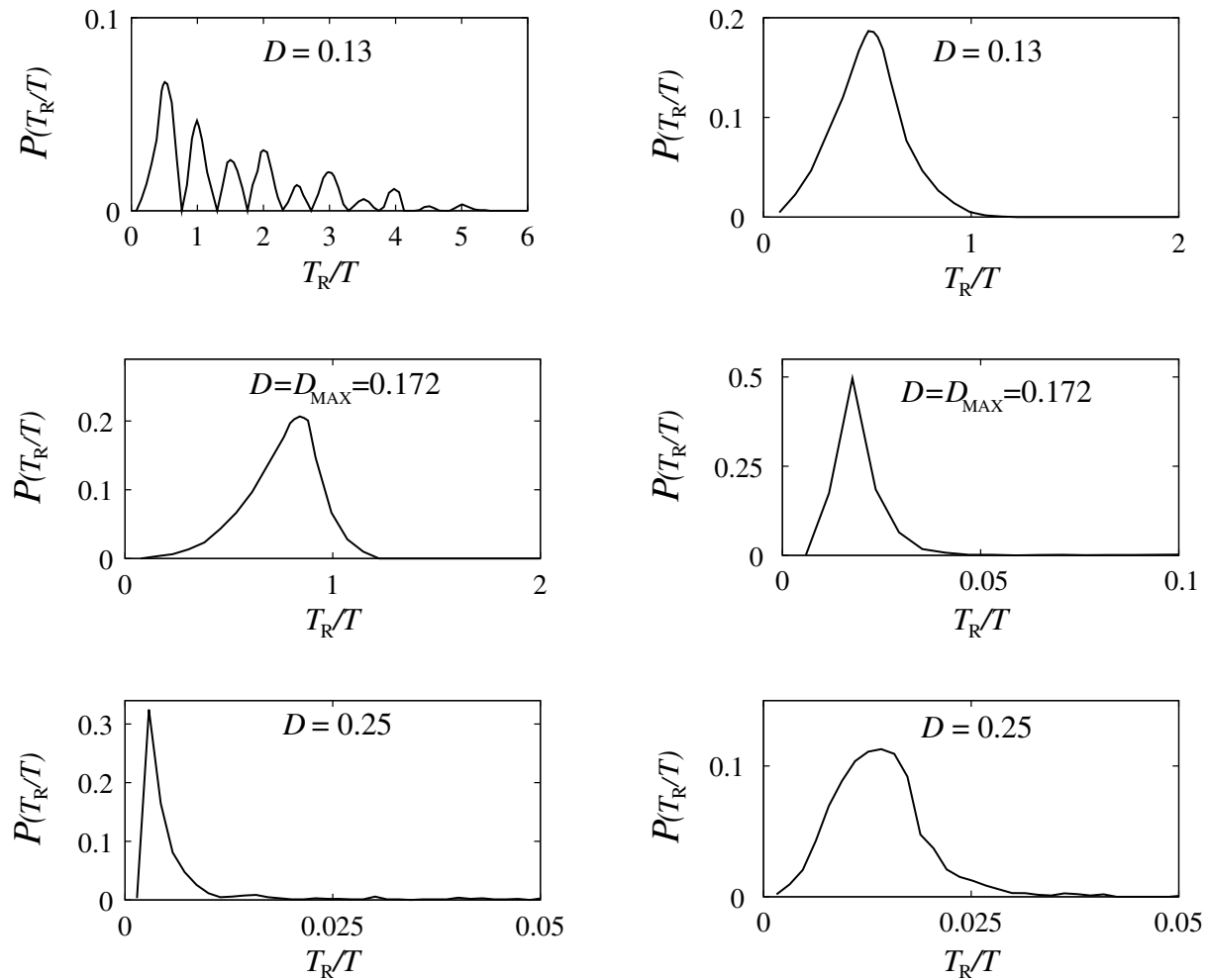

Fig. 10. Same as Fig. 8 but for the asymmetric system 1 with $\alpha=300$. 
at $T_{\mathrm{R}} / T=n, n=1,2, \ldots$ This can be understood. For example, for $\alpha=0.02$ the depth of the left-well is considerably lower than that of $\alpha=1$. As a result, the particle in the left-well will be able to pass to the right-well even when the left-well depth is about maximum. If this is the case, then at this time the right-well depth is about minimum. Since $V(x)$ for $x>0$ is independent of $\alpha$ value, the chance for the particle in the right-well is higher when the depth of the right-well is about minimum. This will happen after integral multiples of period- $T$ of the external force. As a result, we expect peaks in $P\left(T_{\mathrm{R}} / T\right)$ about $T_{\mathrm{R}} / T=1,2, \ldots$ and are seen in Fig. 9 for $\alpha=0.02$ and $D=0.08$. At $D=D_{\mathrm{MAX}}=0.11$

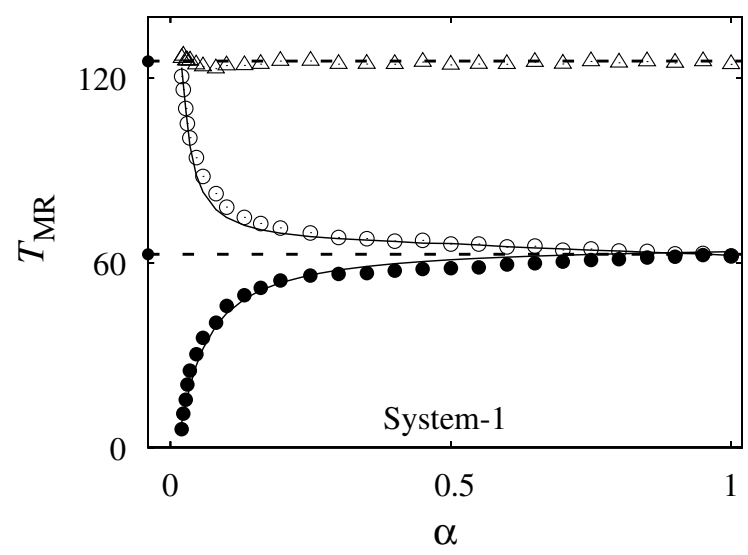

(a)

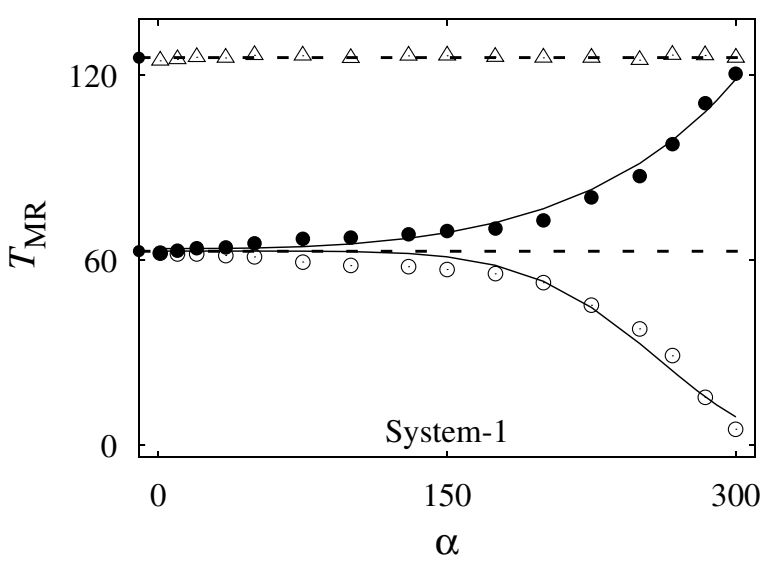

(b)

Fig. 11. Mean residence time $T_{\mathrm{MR}}$ of the left-well (marked by $\bullet$ ) and the right-well (marked by $\circ$ ) versus $\alpha$ for system 1 . Continuous lines are the best curve fit. For clarity, the period $T=125.66 \ldots$ and the half period $T / 2=62.83 \ldots$ of the periodic input signal are represented by dashed lines. The symbol $\triangle$ represents the sum of numerically calculated $T_{\mathrm{MR}}^{\mathrm{L}}$ and $T_{\mathrm{MR}}^{\mathrm{R}}$. For each value of $\alpha$ the noise intensity $D$ is fixed at $D_{\text {MAX }}$ where SNR is maximum. the $P\left(T_{\mathrm{R}} / T\right)$ of both wells have only a single dominant peak and the corresponding $T_{\mathrm{R}} / T$ values of the left- and right-wells are different. That is, the mean residence times of both wells are different. The characteristic properties of the left-well and the right-well are interchanged for $\alpha>1$ compared to $\alpha<1$ (Figs. 9 and 10). For $D \gg D_{\mathrm{MAX}}$, for both $\alpha<1$ and $\alpha>1$, the distribution is too narrow in both wells. The reason is that for large values of noise intensity the motion is dominated by the noise term and a very rapid switching between both wells occurs. The above type of distinct patterns of $P\left(T_{\mathrm{R}} / T\right)$ in both wells is observed in the two other asymmetric systems as well.

Finally, we point out the effect of asymmetries on the mean residence time $\left(T_{\mathrm{MR}}\right)$. As seen in Figs. $7(\mathrm{~b})-7(\mathrm{~g})$, the asymmetric systems for each fixed value of $\alpha$ at $D=D_{\mathrm{MAX}}$ undergo periodic switching between both wells but with different periodicity for the right-well and the left-well. We numerically calculate $T_{\mathrm{MR}}$ in each well by averaging over a set of $10^{5}$ residence times at $D_{\text {MAX }}$. When $\alpha=0.02$, the mean residence time in the left-well of system 1 is $T_{\mathrm{MR}}^{\mathrm{L}}=18.92$ and that of the right-well is $T_{\mathrm{MR}}^{\mathrm{R}}=106.06$. Even though, $T_{\mathrm{MR}}^{\mathrm{L}} \neq T_{\mathrm{MR}}^{\mathrm{R}}$, we notice that $T_{\mathrm{MR}}^{\mathrm{L}}+T_{\mathrm{MR}}^{\mathrm{R}} \approx T$. For $\alpha=300, T_{\mathrm{MR}}^{\mathrm{L}}=120.32, T_{\mathrm{MR}}^{\mathrm{R}}=5.12$ and here again $T_{\mathrm{MR}}^{\mathrm{L}}+T_{\mathrm{MR}}^{\mathrm{R}} \approx T$. In the other two asymmetric systems, the above characteristic of $T_{\mathrm{MR}}^{\mathrm{L}}$ and $T_{\mathrm{MR}}^{\mathrm{R}}$ is found too. In system 1 , as shown in Fig. 11(a) as $\alpha$ decreases from $\alpha=1, T_{\mathrm{MR}}^{\mathrm{L}}\left(T_{\mathrm{MR}}^{\mathrm{R}}\right)$ decreases (increases) from $T / 2$. The opposite of this is observed when $\alpha$ is increased from 1 [Fig. 11(b)]. For $\alpha \in[0.5,100]$ the deviation of $T_{\mathrm{MR}}^{\mathrm{L}}$ and $T_{\mathrm{MR}}^{\mathrm{R}}$ from $T / 2$ is very small. Rapid variation of them are found for $\alpha>100$ and $\alpha \ll 1$. In systems 2 and $3 T_{\mathrm{MR}}^{\mathrm{L}}$ and $T_{\mathrm{MR}}^{\mathrm{R}}$ also vary with $\alpha$ (Fig. 12 for

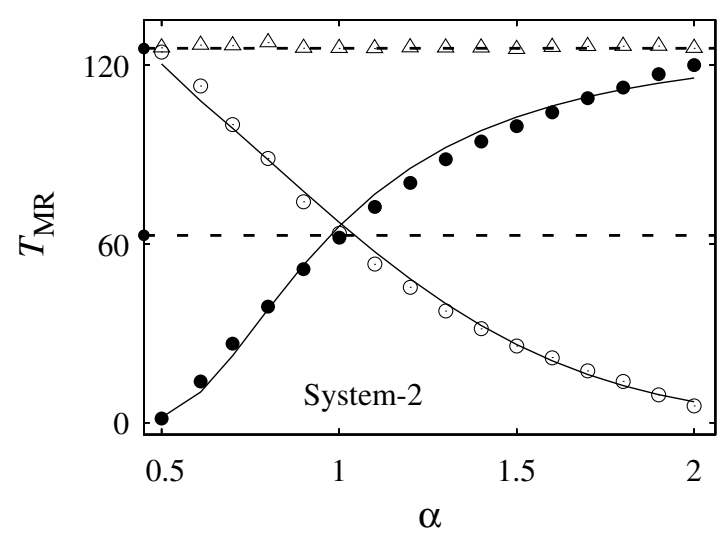

Fig. 12. Same as in Fig. 11 but for system 2 . 
system 2 ) and both become $T / 2$ when $\alpha=1$. In system $2, T_{\mathrm{MR}}^{\mathrm{L}} \approx 172 \mathrm{e}^{-1 / \alpha^{2}}$ and $T_{\mathrm{MR}}^{\mathrm{R}} \approx 172 \mathrm{e}^{-\alpha^{2}}$. In system $3 T_{\mathrm{MR}}^{\mathrm{L}} \approx 171 \mathrm{e}^{-1 / \alpha}$ and $T_{\mathrm{MR}}^{\mathrm{R}} \approx 171 \mathrm{e}^{-\alpha}$. We note that the value of the scaling exponent in the above fits is 1 .

\section{Conclusion}

To conclude, we have shown the influence of three types of asymmetries in the double-well Duffing oscillator system on $\mathrm{SR}$. In all three systems $D_{\mathrm{MAX}}$ increases with increase in $\alpha$ and is proved numerically. On the other hand, $\mathrm{SNR}_{\mathrm{MAx}}$ increases with increase in $\alpha$ in systems 1 and 3 but the reverse effect is observed in system 2 . In the symmetric system $T_{\mathrm{MR}}^{\mathrm{L}}=T_{\mathrm{MR}}^{\mathrm{R}}=T / 2$ but in the asymmetric systems $T_{\mathrm{MR}}^{\mathrm{L}} \neq T_{\mathrm{MR}}^{\mathrm{R}}$, however $T_{\mathrm{MR}}^{\mathrm{L}}+T_{\mathrm{MR}}^{\mathrm{R}} \approx T$. It is evident from the probability distribution of the normalized residence time of the left-well and the right-well that, at $D=D_{\mathrm{MAX}}$ the $P\left(T_{\mathrm{R}} / T\right)$ of each well has a dominant peak at the mean residence time and the location of the dominant peak is different for both wells in the asymmetrical systems. There are asymmetric wave forms, such as symmetric saw-tooth wave, rectified sine wave, rectangular waves, variable shape force, etc. which can be generated easily and they have practical applications. Their periodicity, amplitude and asymmetry are rather easy to control. Our study indicates that asymmetries in the external force and noise may also have a strong influence on the characteristics of SR. The study of SR with asymmetric force and noises will provide much insight on the scaling of SNR and mean residence time and such analysis can be performed analytically and experimentally. These results are also important for an optimum design of corresponding engineering applications.

\section{References}

Borromeo, M. \& Marchesoni, F. [2007] "Stochastic synchronization via noise recycling," Phys. Rev. E 75, 041106:1-5.

Borromeo, M. \& Marchesoni, F. [2010] "Double stochastic resonance over an asymmetric barrier," Phys. Rev. E 81, 012102:1-4.
Evstigneev, M., Reimann, P., Pankov, V. \& Prince, R. H. [2004] "Stochastic resonance in monostable overdamped systems," Europhys. Lett. 65, 7-12.

Gammaitoni, L., Marchesoni, F., MenichellaSaetta, E. \& Santucci, S. [1989] "Stochastic resonance in bistable systems," Phys. Rev. Lett. 62, 349-352.

Gammaitoni, L., Hänggi, P., Jung, P. \& Marchesoni, F. [1998] "Stochastic resonance," Rev. Mod. Phys. 70, 223-287.

Gomes, I., Mirasso, C. R., Toral, R. \& Calvo, O. [2003] "Experimental study of high frequency stochastic resonance in Chua circuits," Physica A 327, 115-119.

Jung, P. [1993] "Periodically driven stochastic systems," Phys. Rep. 234, 175-295.

Li, J. [2002] "Effect of asymmetry on stochastic resonance and stochastic resonance induced by multiplicative noise and by mean-field coupling," Phys. Rev. E 66, 031104:1-7.

Marchesoni, F., Apostolico, F. \& Santucci, S. [1999] "Stochastic resonance in an asymmetric Schmitt trigger," Phys. Rev. E 59, 3958-3963.

Masoller, C. [2002] "Noise-induced resonance in delayed feedback systems," Phys. Rev. Lett. 88, 034102:1-4.

McNamara, B. \& Wiesenfeld, K. [1989] "Theory of stochastic resonance," Phys. Rev. A 39, 4854-4869.

Moon, F. C. \& Holmes, P. J. [1979] "A magnetoelastic strange attractor," J. Sound Vibr. 65, 275-296.

Mueller, F., Heugel, S. \& Wang, L. J. [2009] "Optomechanical stochastic resonance in a macroscopic torsion oscillator," Phys. Rev. A 79, 031804(R):1-4.

Simiu, E. [2002] Chaotic Transitions in Deterministic and Stochastic Dynamical Systems (Princeton University Press, NJ).

So, F. \& Liu, K. L. [2002] "Coherent stochastic resonance in a sextic double-well potential," Physica A 303, 79-90.

Stokes, N. G., Stein, N. D. \& McClintock, P. V. E. [1993] "Stochastic resonance in monostable systems," J. Phys. A 26, L385-L390.

Wio, H. S. \& Bouzat, S. [1999] "Effect of asymmetry on stochastic resonance and stochastic resonance induced by multiplicative noise and by mean-field coupling," Braz. J. Phys 29, 136-143.

Zimmerman, N. M., Golding, B. \& Haemmerle, W. H. [1991] "Magnetic field tuned energy of a single twolevel system in a meso-scopic metal," Phys. Rev. Lett. 67, 1322-1325. 